\title{
The Induction with Foley OR Misoprostol (INFORM) Study dataset. A dataset of 602 women with hypertensive disease in pregnancy, in India, randomised to either Foley catheter or oral misoprostol for induction of labour
}

Shuchita Mundle ${ }^{1}$, Hillary Bracken², Vaishali Khedikar ${ }^{3}$, Jayashree Mulik4 ${ }^{4}$, Brian Faragher ${ }^{5}$, Thomas Easterling ${ }^{6}$, Simon Leigh ${ }^{7}$, Paul Granby ${ }^{8}$, Alan Haycox ${ }^{9}$, Mark A. Turner ${ }^{10}$, Kate Lightly ${ }^{10}$, Miroslava Ebringer ${ }^{10}$,

Zarko Alfirevic ${ }^{10}$, Beverly Winikoff ${ }^{11}$ and Andrew D. Weeks ${ }^{10^{*}}$ (i)

\begin{abstract}
Objectives: Induction of labour (IOL), or starting labour artificially, can be a lifesaving intervention for pregnant women and their babies, and rates are rising significantly globally. As rates increase, it becomes increasingly important to fully evaluate all available data, especially that from low income settings where the potential benefits and harms are greater. The goal of this paper is to describe the datasets collected as part of the Induction with Foley OR Misoprostol (INFORM) Study, a randomised trial comparing two of the recommended methods of cervical ripening for labour induction, oral misoprostol and Foley catheter, in women being induced for hypertension in pregnancy, at two sites in India during 2013-15.

Data description: This dataset includes comprehensive data on 602 women who underwent IOL for hypertensive disorders in pregnancy. Women were randomly assigned to cervical ripening with oral misoprostol or a transcervical Foley catheter in two government hospitals in India. The main dataset has 367 variables including monitoring during the induction of labour, medications administered, timing and mode of delivery, measures of neonatal morbidity and mortality, maternal mortality and morbidity, maternal satisfaction and health economic data. The dataset is anonymised and available on ReShare.
\end{abstract}

Keywords: Induction, Labour, Hypertension, Pre-eclampsia, Misoprostol, Foley catheter, Dataset

\section{Objectives}

IOL rates are rising rapidly around the world, both in high and low income settings. High quality IOL studies

\footnotetext{
${ }^{*}$ Correspondence: aweeks@liverpool.ac.uk

${ }^{10}$ University of Liverpool and Liverpool Women's Hospital for Liverpool Health Partners, Crown Street, Liverpool L8 7SS, UK

Full list of author information is available at the end of the article

The study protocol for this work was published in BMC Pregnancy and childbirth. https://doi.org/10.1186/1471-2393-14-308.
}

from low income settings are rare, and most are undertaken in high income settings. However, the findings of high income setting studies may not be applicable to resource constrained settings where the risks and benefits of induction are far higher for patients, and adequate resources are often not available. Pre-eclampsia is one of the most prevalent causes of morbidity and mortality around the world. This dataset, which also includes health economic data, is important for researchers, 
clinicians and policy makers investigating IOL, maternity care in high and low income settings and pre-eclampsia.

Low dose oral misoprostol and the Foley catheter are low cost cervical ripening methods recommended for use around the world including low income settings, where pre-eclampsia causes the most significant burden [1]. However few studies have directly compared them [2, 3]. Few similar datasets of pre-eclamptic patients and IOL patients are currently available. The INFORM study was undertaken at two public hospitals in Nagpur, India, between December 2013 and June 2015. The aims of this randomised controlled trial were "to directly compare the efficacy, safety, acceptability and cost effectiveness of misoprostol and the Foley catheter in the induction of labour among women with gestational hypertension, in a low resource setting" $[4,5]$.

The study protocol [4], clinical results [5] and health economic analysis [6] have been published previously, but the dataset was not published at that time due to concerns that some variables could potentially compromise patient and/or research staff confidentiality; these issues have now been resolved as described later in this paper.

\section{Data description}

Women included in this study were at least 18 years old, over 20 weeks' gestation, with a live fetus and were scheduled to have an induced labour because of hypertensive disorders of pregnancy. Women unable to give informed consent, those with a previous caesarean section, multiple pregnancy, ruptured membranes, clinically diagnosed chorioamnionitis or a history of allergy to misoprostol were not recruited [4].

After informed consent, 602 women were randomly assigned to labour induction with oral misoprostol ( $25 \mu$ g every $2 \mathrm{~h}$ for a maximum of 12 doses) or a transcervical Foley catheter (size $18 \mathrm{~F}$ with $30 \mathrm{ml}$ balloon). Randomisation schedules were computer-generated and administered using opaque sealed envelopes. Induction continued with artificial membrane rupture and oxytocin, administered through a micro-drip gravity infusion set [4].

Detailed information was collected on paper forms by research assistants at defined time points; immediately prior to IOL, at randomisation, every $2 \mathrm{~h}$ during the IOL process, at $24 \mathrm{~h}$ post-delivery and at discharge. Data were later double-entered into SPSS (IBM, Portsmouth, UK) by research staff in India and the US. The case report forms have been made publicly available along with a data dictionary and detailed description of methods (see Table 1).

Data on admission, induction and delivery includes basic demographic information, medical and obstetric history (current and previous), pre-eclampsia symptoms (severe nausea and vomiting, epigastric pain, headache, visual disturbance, chest pain or dyspnea), maternal and fetal observations and examination findings, detailed information on all medication administered, women's expectations of pain and anxiety pre-IOL, side effects and complications of induction, mode of delivery and indication for delivery where relevant, complications and operative interventions. These data were used to calculate the number of vaginal births within specific timeframes (12, $24 \mathrm{~h}$ ) and induction to birth intervals.

Postnatal data includes information on morbidity, complications, mortality, women's rating of acceptability (regarding the amount of time taken and anxiety), whether women would recommend this method for future inductions, discharge dates and discharge medications.

Neonatal data include basic information such as birth outcome, birth weight, APGARs at 1, 5 and $10 \mathrm{~min}$, age of first feed, gasp and heart rate over 100 . Neonatal morbidity including diagnosis, SCBU admission and length of stay, oxygen administration, ventilation, seizures and age at first seizure, discharge/death date and discharge medication. Upon discharge, babies admitted to the special care unit were assessed for encephalopathy and the components of the original Sarnat score recorded [4].

Table 1 Overview of data files/data sets

\begin{tabular}{|c|c|c|c|}
\hline Label & Name of data file/data set & $\begin{array}{l}\text { File types } \\
\text { (file extension) }\end{array}$ & Data repository and identifier (DOI or accession number) \\
\hline Data file 1 & INFORM database_CSV_160121 & CSV data file (.csv) & $\begin{array}{l}\text { UK Data Service ReShare http://doi.org/10.5255/UKDA-SN- } \\
854663 \text { [7] }\end{array}$ \\
\hline Data file 2 & INFORM_database_Excel_060121 & MS Excel file (.x|sx) & $\begin{array}{l}\text { UK Data Service ReShare http://doi.org/10.5255/UKDA-SN- } \\
854663 \text { [7] }\end{array}$ \\
\hline Data file 3 & INFORM_Data Dictionary_160121_sharing version & MS Excel file (.xlsx) & $\begin{array}{l}\text { UK Data Service ReShare http://doi.org/10.5255/UKDA-SN- } \\
854663 \text { [7] }\end{array}$ \\
\hline Data file 4 & Detailed_methods_INFORM_16NOV20.docx & MS Word file (.docx) & $\begin{array}{l}\text { UK Data Service ReShare http://doi.org/10.5255/UKDA-SN- } \\
854663 \text { [7] }\end{array}$ \\
\hline
\end{tabular}


Full details of the outcomes collected are included in the downloadable data dictionary and case report forms (see Table 1).

Due to concerns that including explicit dates (particularly date of delivery) could compromise anonymity, all dates have been replaced by number of days from a seed date prior to the start of this study; the identity of the seed date can be obtained on request, where appropriate.

\section{Limitations}

This was a pragmatic study, based in busy Government Hospitals, where clinical decisions are typically made quickly, often without the full diagnostic work-ups and investigations required to differentiate between subgroups of hypertension in pregnancy (i.e. separation into proteinuric or non-proteinuric hypertension, or HELLP syndrome). Urinalysis was often not available, therefore making these results more generalizable to the whole population, but less focused on the treatment of hypertensive disorders specifically. Further investigations such as blood tests were not recorded.

This was an un-blinded study, due to ethical and practical concerns associated with undertaking unnecessary or sham Foley catheterisation. Whilst this risks bias, neither treatment was commonly used in the recruiting hospitals prior to this study and therefore clinician's pre-existing views about treatment efficacy were unlikely to affect clinical decisions on these induction methods.

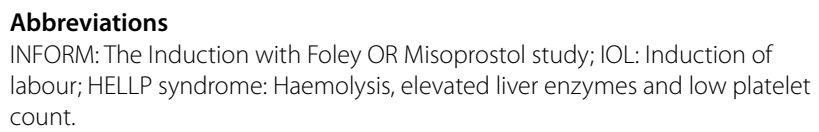

\section{Acknowledgements}

We are so grateful to all of the patients, clinicians, research associates, trial management committee members, administrative and clinical trial support staff who contributed to this valuable work.

\section{Authors' contributions \\ ADW had the original idea for the study and is guarantor for the study. The idea was then developed into a formal grant application with SM, BW, HB, ZA, $\mathrm{BF}, \mathrm{TE}$, and $\mathrm{AH}$. SM led the study team in India, with the principle investigators VK and JM ensuring accurate and valid data acquisition from the study sites. SL and $\mathrm{PG}$ joined $\mathrm{AH}$ to conduct the economic analysis, and MT joined to provide academic neonatal support. SM, HB, BF, SL, BW and ADW formed the trial management team with input from other co-investigators as required. HB was the study monitor. BF prepared the data for sharing and conducted the main analysis and ADW wrote the first draft of the clinical paper. $\mathrm{KL}$ and ME wrote the first draft of this data paper, using text from the previous publications. SL prepared the health economic data. All authors reviewed and accepted the paper prior to submission.}

\section{Funding}

The trial was funded by DFID/MRC/Wellcome Trust through the Joint Global Health Trials Scheme (ref G1 100686/1), to the University of Liverpool. The funder had no involvement in the data collection, running of this trial or contribution to the publications. The funder had a representative on the independent trial steering committee and approved its membership.

\section{Availability of data and materials}

The data described in this data note can be accessed on UK Data Service's ReShare data repository under http://doi.org/10.5255/UKDA-SN-854663. Please see Table 1 and reference list [7] for details and links to the data.

\section{Declarations}

Ethics approval and consent to participate

The Research Ethics Committees at Government Medical College, Nagpur and the University of Liverpool both approved this study. Women provided written and video-recorded oral consent, as per the Drug Controller General of India recommendations, at that time. Study monitoring was undertaken by an independent trial steering committee and data monitoring committee. The trial was registered on ClinicalTrials.gov (NCT01801410).

\section{Consent for publication}

The INFORM consent form allowed women to tick an extra box confirming that they were agreeable for their data to be used for future research studies. All women recruited to the INFORM study consented to share their data.

\section{Competing interests}

ADW is a Scientific Advisor to Norgine, a Dutch pharmaceutical company. The company pays the University of Liverpool for his time and he receives no personal payments. MAT has provided consultancy services to Chiesi, BMS, Novartis, Shire, Janssen \& Grunenthal. The companies pay the University of Liverpool for his time and he receives no personal payments. There are no other conflicts of interest to declare.

\section{Author details}

${ }^{1}$ Department of Obstetrics and Gynecology, All India Institute of Medical Sciences, Nagpur, India. ${ }^{2}$ Gynuity Health Projects (GHP), 220 East 42nd Street, New York, NY 10017, USA. ${ }^{3}$ Daga Memorial Women's Government Hospital, Nagpur, India 440018. ${ }^{4}$ Government Medical College, Nagpur 440003, India. ${ }^{5}$ Liverpool School of Tropical Medicine, Pembroke Place, Liverpool L3 5QA, UK. ${ }^{6}$ University of Washington, Seattle, Washington 98195, USA. ${ }^{7}$ Nexus Clinical Analytics, Euxton PR7 6BX, Lancashire, UK. ${ }^{8}$ Certus Analytics, Formby, Liverpool L37 2LR, UK. ${ }^{9}$ University of Liverpool Management School, Chatham Street, Liverpool L69 7ZH, UK. ${ }^{10}$ University of Liverpool and Liverpool Women's Hospital for Liverpool Health Partners, Crown Street, Liverpool L8 7SS, UK.

${ }^{11}$ Gynuity Health Projects (GHP), 220 East 42nd Street, New York, NY 10017, USA.

Received: 13 April 2021 Accepted: 1 September 2021

Published online: 10 September 2021

\section{References}

1. WHO. WHO recommendations for induction of labour. Geneva: World Health Organization; 2011.

2. Ten Eikelder ML, Oude Rengerink K, Jozwiak M, et al. Induction of labour at term with oral misoprostol versus a Foley catheter (PROBAATII): a multicentre randomised controlled non-inferiority trial. Lancet. 2016;387:1619-28.

3. Ten Eikelder ML, van de Meent MM, Mast K, et al. Women's experiences with and preference for induction of labor with oral misoprostol or Foley catheter at term. Am J Perinatol. 2017;34:138-46.

4. Bracken $H$, Mundle $S$, Faragher $B$, et al. Induction of labour in pre-eclamptic women: a randomised trial comparing the Foley balloon catheter with oral misoprostol. BMC Pregnancy Childbirth. 2014;14:308.

5. Mundle S, Bracken H, Khedikar $\mathrm{V}$, et al. Foley catheterisation versus oral misoprostol for induction of labour in hypertensive women in India (INFORM): a multicentre, open-label, randomised controlled trial. Lancet. 2017;390(10095):669-80. https://doi.org/10.1016/S0140-6736(17) 31367-3.

6. Leigh S, Granby P, Haycox A, et al. Foley catheter vs. oral misoprostol to induce labour among hypertensive women in India: a cost-consequence 
analysis alongside a clinical trial. BJOG. 2018;125(13):1734-42. https://doi. org/10.1111/1471-0528.15285.

7. Mundle S, Bracken H, Khedikar V, Mulik J, Faragher B, Easterling T, et al. The Induction with Foley OR Misoprostol (INFORM) Study, 2013-2015.

[Data Collection]. UK data service. 2021. https://doi.org/10.5255/

UKDA-SN-854663

\section{Publisher's Note}

Springer Nature remains neutral with regard to jurisdictional claims in published maps and institutional affiliations.
Ready to submit your research? Choose BMC and benefit from:

- fast, convenient online submission

- thorough peer review by experienced researchers in your field

- rapid publication on acceptance

- support for research data, including large and complex data types

- gold Open Access which fosters wider collaboration and increased citations

- maximum visibility for your research: over $100 \mathrm{M}$ website views per year

At BMC, research is always in progress.

Learn more biomedcentral.com/submissions 